\section{Rare and Fatal Gastrointestinal Mucormycosis (Zygomycosis) in a COVID-19 Patient: A Case Report}

\author{
Epifanio Silvino do Monte Junior, Marcos Eduardo Lera dos Santos, Igor Braga Ribeiro, Gustavo de Oliveira Luz, Elisa Ryoka \\ Baba, Bruno Salomão Hirsch, Mateus Pereira Funari and Eduardo Guimarães Hourneaux de Moura
}

Gastrointestinal Endoscopy Unit, Hospital das Clínicas da Faculdade de Medicina da Universidade de São Paulo, São Paulo, Brasil

The novel coronavirus disease (COVID-19) quickly spread to all continents. However, data regarding all the signs and symptoms of COVID-19 are insufficient. Patients with COVID-19 might present higher susceptibility to fungal coinfections. Mucormycosis is a rare and often life-threatening fungal disease characterized by vascular invasion by hyphae, resulting in thrombosis and necrosis. This is the first case report of mucormycosis in a COVID-19 patient. An 86-year-old male patient was admitted to the emergency room with acute diarrhea, cough, dyspnea, and fever from 5 days prior. Blood tests revealed a hemoglobin level of $14.3 \mathrm{mg} / \mathrm{dL}$. Five days following the admission, the patient presented with melena and a hemoglobin level of $5.6 \mathrm{mg} / \mathrm{dL}$. A transfusion of three units of red blood cells was required. Esophagogastroduodenoscopy revealed two giant gastric ulcers with necrotic debris and a deep hemorrhagic base without active bleeding. Furthermore, biopsies confirmed mucormycosis. Despite intensive care, the patient died 36 hours after the esophagogastroduodenoscopy. Clin Endosc 2020;53:746-749

Key Words: Coronavirus infections; Gastroenteritis; Gastrointestinal hemorrhage; Mucormycosis; Zygomycosis

\section{INTRODUCTION}

Mucormycosis (previously called zygomycosis) is a rare and devastating disease caused by a ubiquitous fungus that belongs to the class Zygomycetes and order Mucorales. ${ }^{1}$ The clinical presentation of mucormycosis varies depending on the location of the disease. ${ }^{2}$ The majority of patients with invasive mucormycosis are either undergoing treatment with immunosuppressants or have underlying conditions, such as diabetes mellitus, hematologic malignancies, and trauma or are transplant recipients. Outbreaks of the fungal disease have also been associated with natural disasters. ${ }^{3-5}$

Received: July 7, 2020 Revised: August 19, 2020

Accepted: September 5, 2020

Correspondence: Igor Braga Ribeiro

Gastrointestinal Endoscopy Unit, Hospital das Clínicas da Faculdade de Medicina da Universidade de São Paulo, Av. Dr Enéas de Carvalho Aguiar, 225, $6^{\circ}$ Andar, Bloco 3, Cerqueira Cesar, São Paulo 05403-010, Brasil

Tel: +55-92-98137-7788, Fax: +55-11-2661-6467, E-mail: igorbraga1@gmail.com ORCID: https://orcid.org/0000-0003-1844-8973

(c) This is an Open Access article distributed under the terms of the Creative Commons Attribution Non-Commercial License (http://creativecommons.org/ licenses/by-nc/3.0) which permits unrestricted non-commercial use, distribution, and reproduction in any medium, provided the original work is properly cited.
Although unusual, mucormycosis of the gastrointestinal (GI) tract occurs as a result of the ingestion of the spores of the fungus. Cases of GI mucormycosis in an immunocompetent host are rarely reported; however, the mortality rate due to GI mucormycosis can be as high as $85 \%{ }^{6}$

In early December 2019, the first cases of severe acute respiratory syndrome coronavirus 2 (SARS-CoV-2) infection were identified in Wuhan. ${ }^{7,8}$ As this is a relatively novel virus, data regarding all the signs indicative of coronavirus disease (COVID-19) and symptoms that can be caused by the disease are insufficient. ${ }^{9}$ Furthermore, little is known about other diseases that can increase the risk of SARS-CoV-2 coinfection. To the best of our knowledge, this is the first case of mucormycosis reported in a COVID-19 patient.

\section{CASE REPORT}

An 86-year-old male patient with a history of arterial hypertension was admitted to the emergency room with acute diarrhea, cough, dyspnea, and fever that started 5 days before admission. Blood analysis revealed a hemoglobin level of 14.3 
$\mathrm{mg} / \mathrm{dL}$, a leukocyte count of $11.2 \times 109 / \mathrm{L}$, lymphocyte count of $0.58 \times 10^{9} / \mathrm{L}$, a D-dimer level of $602 \mathrm{ng} / \mathrm{mL}$, a lactate dehydrogenase level of $850 \mathrm{U} / \mathrm{L}$, a creatinine level of $2.34 \mathrm{mg} / \mathrm{dL}$, and a urea level of $93 \mathrm{mg} / \mathrm{dL}$. A throat swab was collected from the patient, which confirmed COVID-19. Computed tomography of the chest was performed, and the findings are demonstrated in Fig. 1. Due to acute respiratory failure and hemodynamic instability, he was transferred to the intensive care unit (ICU). The patient was initially treated with ceftriaxone, azithromycin, oseltamivir, and hydrocortisone, besides intensive care management including vasopressors and mechanical ventilation. Five days following ICU admission, the patient presented with melena and severe anemia (hemoglobin level of $5.6 \mathrm{mg} /$ dL). Physical examination revealed mild abdominal tenderness. He was managed with three units of red blood cells and omeprazole. Esophagogastroduodenoscopy (EGD) revealed two giant gastric ulcers with dirty debris and a deep hemorrhagic base without active bleeding located in the greater and lesser curvature (Fig. 2). Pathological examination confirmed mucormycosis (Fig. 3). Unfortunately, the patient died 1 week following hospitalization, 36 hours after the EGD, and before a diagnosis was established. Antifungal agents were not administered.

\section{DISCUSSION}

The ongoing outbreak of COVID-19 originated in Wuhan, China, in December 2019. COVID-19, which is the disease associated with SARS-CoV-2 infection, spread rapidly to produce a global pandemic. ${ }^{10}$ The spectrum of symptomatic SARS-CoV-2 infection ranges from mild to critical. The proportion of severe or fatal infections may also vary by location.

Patients with COVID-19 might present with markedly higher levels of inflammatory cytokines (such as interleukin [IL]-2R, IL-6, IL-10, and tumor necrosis factor-alpha), associated with impaired cell-mediated immune response, affecting both CD $4+\mathrm{T}$ and CD8 $+\mathrm{T}$ cells. Hence, an increased susceptibility to fungal coinfections is observed. ${ }^{11}$

Mucormycosis is characterized by infarction and necrosis of the host tissues that result from an invasion of the vasculature by hyphae. Depending on the anatomic site involved, mucormycosis can present as a variety of different syndromes, including rhino-orbital-cerebral, pulmonary, cutaneous, and less often GI, renal, and disseminated diseases. ${ }^{12} \mathrm{GI}$ involvement presents in approximately $8 \%$ of the cases. The most commonly affected organs in the GI tract include the stomach and colon.

The symptoms of GI mucormycosis may include fever, nausea, abdominal pain, GI bleeding, and perforation. Establish-

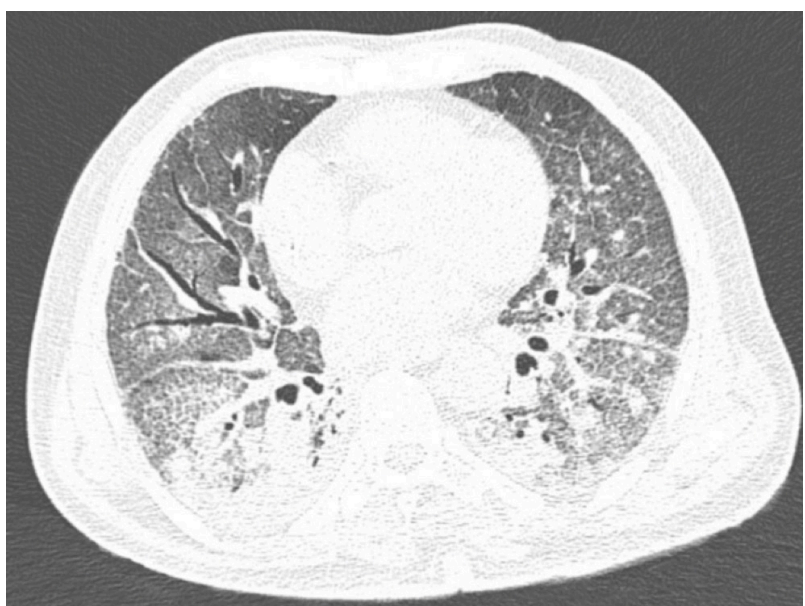

Fig. 1. The chest computed tomography revealed ground-glass opacity with consolidative abnormalities.
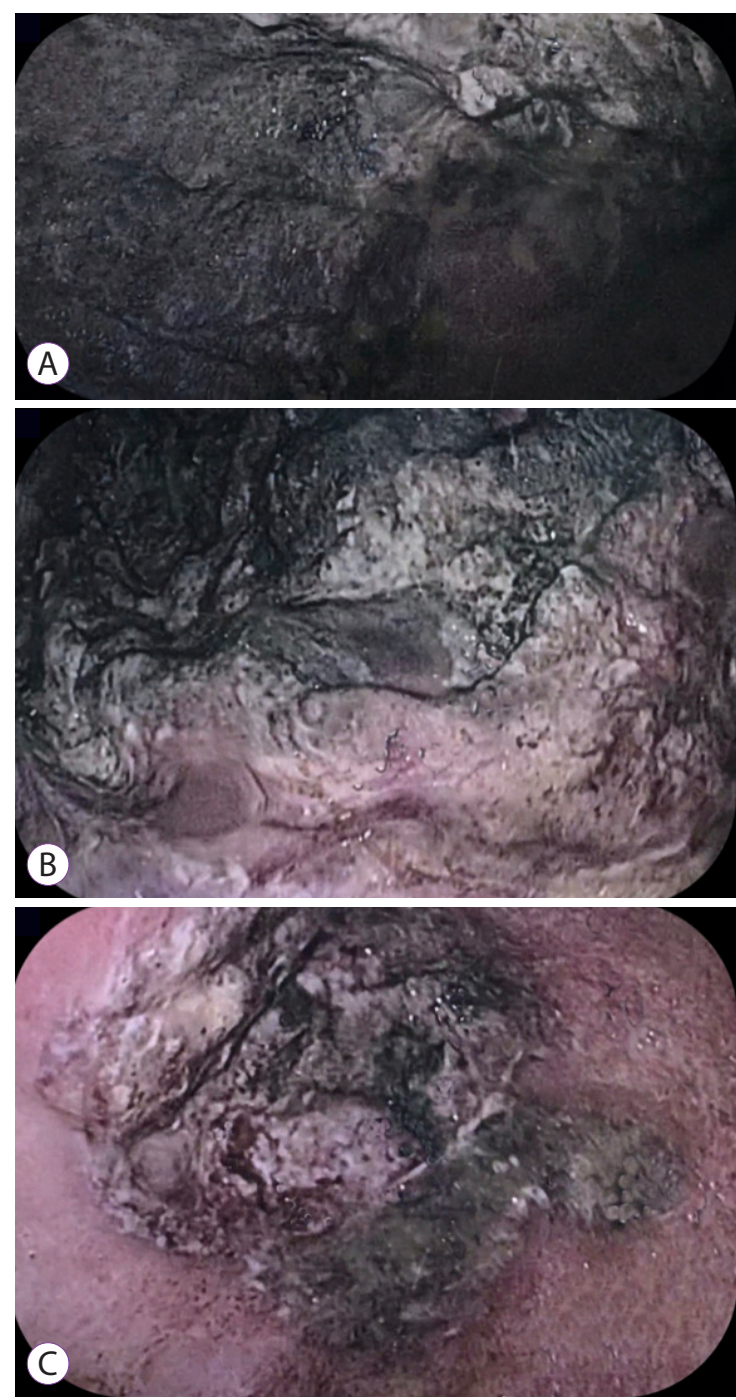

Fig. 2. The esophagogastroduodenoscopy demonstrated a giant ulcer in (A) the greater curvature, (B) fundus, and (C) antrum. 


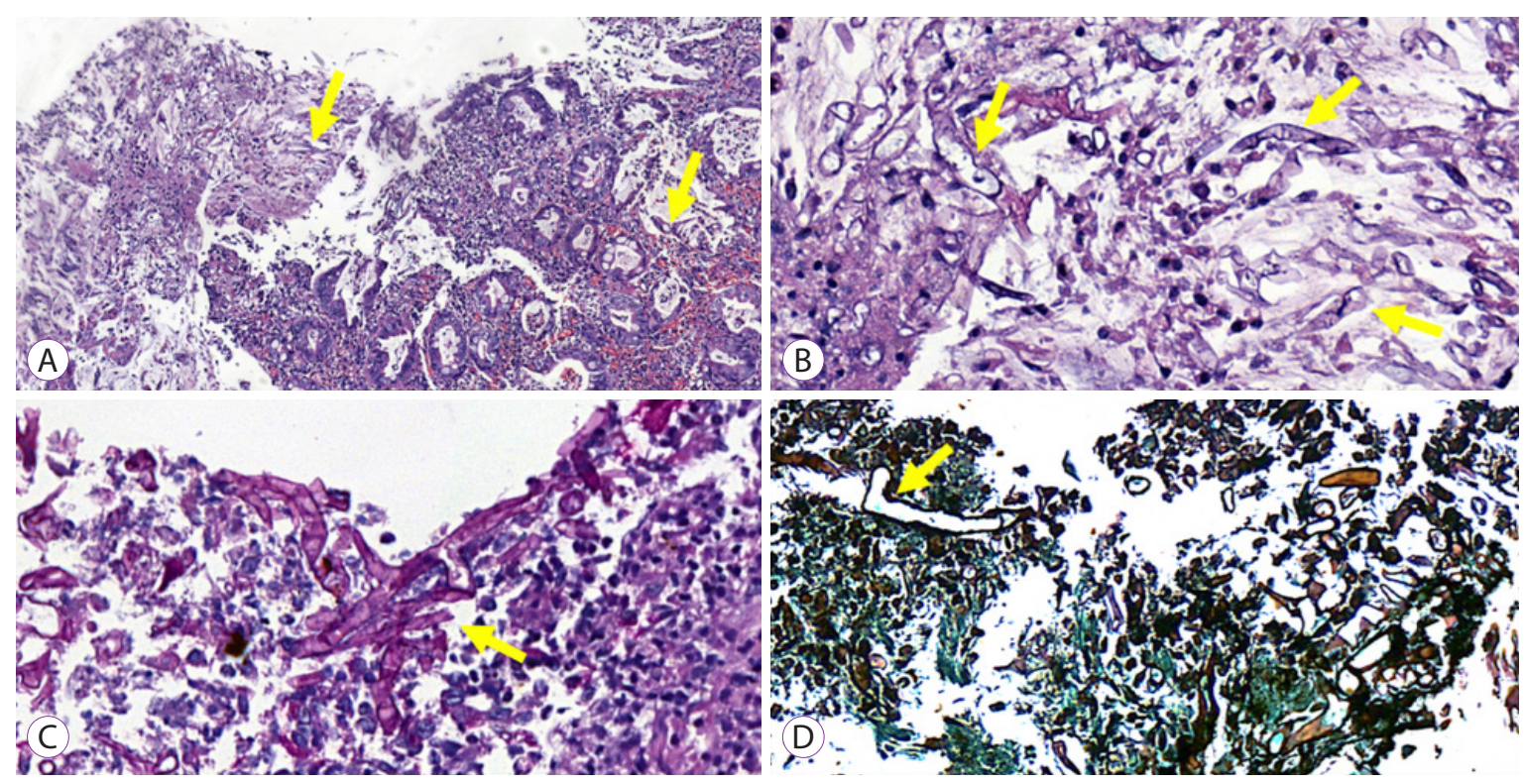

Fig. 3. (A) The gastric border and base with necrotic fibrinoid debris. The fungus structures are visible at a low-power view (arrows). Hematoxylin and eosin staining, original magnification $\times 100$. (B) Presence of broad, irregular, non-septate hyphae of mucormycosis (arrows). Hematoxylin and eosin staining, original magnification $\times 400$. (C) Hyphae stained with periodic acid-Schiff stain (arrow), original magnification $\times 400$. (D) Note the hyphae with typical 90-degree angle branching (arrow). Grocott's methenamine staining, original magnification $\times 400$.

ing a correct diagnosis is challenging due to the nonspecific clinical signs/symptoms, which may not result in suspicions of mucormycosis. The endoscopic appearance of gastric mucormycosis is usually a large ulcer with necrosis, eventually presenting an adherent, thick, green exudate. ${ }^{13,14}$ Diagnosis is confirmed by histopathologic identification based on the biopsy of the suspected area during surgery or endoscopy. ${ }^{6}$ The disease has a high fatality rate (up to $85 \%$ ) related to late or no diagnosis. $^{15}$

Treatment involves surgical debridement of involved tissues and antifungal therapy. Intravenous amphotericin B (a lipid formulation) is the drug of choice for initial therapy. ${ }^{16}$ Despite early diagnosis and aggressive combined surgical and medical therapy, the prognosis for recovery from mucormycosis is poor. ${ }^{6}$

We presented a case of an elderly patient whose only known comorbidity was arterial hypertension, without traditional risk factors for developing mucormycosis. He was admitted following the diagnosis of COVID-19 and acute respiratory failure and managed with broad-spectrum antibiotics and corticosteroids (which are risk factors for invasive fungal disease). ${ }^{17}$ It is known that COVID-19 may be associated with immune dysregulation, ${ }^{18}$ and it has been reported that patients with COVID-19 might be at risk of developing invasive fungal infections, such as invasive aspergillosis, candidiasis, and Pneumocystis jiroveci infection. ${ }^{11,19}$ However, there are no reports of mucormycosis associated with COVID-19. Our patient was diagnosed with primary gastric mucormycosis, but it is unknown whether the cause of death was invasive mucormycosis.

GI mucormycosis is a rare disease, and it should be considered if an atypical gastric ulcer is identified in a COVID-19 patient. Clinical suspicion and prompt treatment are fundamental to achieve the cure of the disease. Hence, preemptive therapy should be considered in the presence of features suggesting mucormycosis. More studies are necessary to determine if these two pathologies are related.

\section{Conflicts of Interest}

Eduardo Guimarães Hourneaux de Moura reports personal fees from Boston Scientific and Olympus outside the submitted work. The other authors have no financial conflicts of interest.

\section{Author Contributions}

Conceptualization: Epifanio Silvino do Monte Junior, Igor Braga Ribeiro Data curation: ESM]

Formal analysis: Marcos Eduardo Lera dos Santos, IBR, Gustavo de Oliveira Luz, Elisa Ryoka Baba

Investigation: ESMJ, MELS, Bruno Salomão Hirsch, Mateus Pereira Funari

Supervision: MELS, IBR, GOL, ERB, Eduardo Guimarães Hourneaux de Moura

Writing-original draft: ESMJ

Writing-review\&editing: IBR 
ORCID

Epifanio Silvino do Monte Junior: https://orcid.org/0000-0001-7304-8222 Marcos Eduardo Lera dos Santos: https://orcid.org/0000-0001-9759-3807 Gustavo de Oliveira Luz: https://orcid.org/0000-0001-7396-8440 Elisa Ryoka Baba: https://orcid.org/0000-0001-7261-9054 Bruno Salomão Hirsch: https://orcid.org/0000-0002-0777-0150 Mateus Pereira Funari: https://orcid.org/0000-0002-4828-9320 Eduardo Guimarães Hourneaux de Moura: https://orcid.org/0000-00028023-3722

\section{REFERENCES}

1. Adhikari S, Gautam AR, Paudyal B, Sigdel KR, Basnyat B. Case report: gastric mucormycosis- a rare but important differential diagnosis of upper gastrointestinal bleeding in an area of Helicobacter pylori endemicity. Wellcome Open Res 2019;4:5.

2. Kauffman CA, Malani AN. Zygomycosis: an emerging fungal infection with new options for management. Curr Infect Dis Rep 2007;9:435-440.

3. Farmakiotis D, Kontoyiannis DP. Mucormycoses. Infect Dis Clin North Am 2016;30:143-163.

4. Jeong W, Keighley C, Wolfe R, et al. The epidemiology and clinical manifestations of mucormycosis: a systematic review and meta-analysis of case reports. Clin Microbiol Infect 2019;25:26-34.

5. Cornely OA, Alastruey-Izquierdo A, Arenz D, et al. Global guideline for the diagnosis and management of mucormycosis: an initiative of the European Confederation of Medical Mycology in cooperation with the mycoses study group education and research consortium. Lancet Infect Dis 2019;19:e405-e421.

6. Roden MM, Zaoutis TE, Buchanan WL, et al. Epidemiology and outcome of zygomycosis: a review of 929 reported cases. Clin Infect Dis 2005;41:634-653.

7. Guan WJ, Ni ZY, Hu Y, et al. Clinical characteristics of coronavirus dis- ease 2019 in China. N Engl J Med 2020;382:1708-1720.

8. Rothan HA, Byrareddy SN. The epidemiology and pathogenesis of coronavirus disease (COVID-19) outbreak. J Autoimmun 2020;109:102433.

9. Moura DTH, McCarty TR, Ribeiro IB, et al. Diagnostic characteristics of serological-based COVID-19 testing: a systematic review and meta-analysis. Clinics (Sao Paulo) 2020;75:e2212.

10. Broughton JP, Deng X, Yu G, et al. CRISPR-Cas12-based detection of SARS-CoV-2. Nat Biotechnol 2020;38:870-874.

11. Song G, Liang G, Liu W. Fungal co-infections associated with global COVID-19 pandemic: a clinical and diagnostic perspective from China. Mycopathologia 2020;185:599-606.

12. Riley TT, Muzny CA, Swiatlo E, Legendre DP. Breaking the mold: a review of mucormycosis and current pharmacological treatment options. Ann Pharmacother 2016;50:747-757.

13. Cherney CL, Chutuape A, Fikrig MK. Fatal invasive gastric mucormycosis occurring with emphysematous gastritis: case report and literature review. Am J Gastroenterol 1999;94:252-256.

14. de Moura DTH, Proença IM, McCarty TR, et al. Gastrointestinal manifestations and associated health outcomes of COVID-19: a Brazilian experience from the largest South American public hospital. Clinics (Sao Paulo) 2020;75:e2271.

15. Petrikkos G, Skiada A, Lortholary O, Roilides E, Walsh TJ, Kontoyiannis DP. Epidemiology and clinical manifestations of mucormycosis. Clin Infect Dis 2012;54 Suppl 1:S23-S34.

16. Spellberg B, Walsh TJ, Kontoyiannis DP, Edwards J, Jr., Ibrahim AS. Recent advances in the management of mucormycosis: from bench to bedside. Clin Infect Dis 2009;48:1743-1751.

17. Salehi M, Ahmadikia K, Badali H, Khodavaisy S. Opportunistic fungal infections in the epidemic area of COVID-19: a clinical and diagnostic perspective from Iran. Mycopathologia 2020;185:607-611.

18. Chen G, Wu D, Guo W, et al. Clinical and immunological features of severe and moderate coronavirus disease 2019. J Clin Invest 2020;130:2620-2629.

19. Koehler P, Cornely OA, Böttiger BW, et al. COVID-19 associated pulmonary aspergillosis. Mycoses 2020;63:528-534. 